\title{
SEXUALITÉ DES LARVES DE MOUSTIQUES
}

\author{
Par M. LANGERON
}

Les malariologistes ne paraissent pas s'être préoccupés du sexe des larves de moustiques. Pourtant on recherche la sexualité des adultes et on l'utilise pour établir des statistiques de la fréquence relative des mâles et des femelles aux différentes époques de l'année et dans les différents gîtes où ces insectes s'abritent. De ces statistiques, on a déduit la notion de l'anophélisme résiduel. C'est ainsi que Swellengrebel (1925, p. 181), désigne la persistance des anophèles adultes dans les maisons et les étables après la disparition des gîtes larvaires. L'anophélisme résiduel est caractérisé par l'absence de mâles. En Europe, l'anophélisme résiduel est très marqué en hiver et paraît ne jouer qu'un rôle effacé dans les infections hivernales et vernales. Au contraire, il prend une grande importance sous les tropiques. De plus, l'anophélisme résiduel n'est pas forcément hivernal ; il peut être très accentué en été et rendu responsable du paludisme automnal et des épidémies de fièvre quarte qui apparaissent en octobre.

La disparition des mâles peut avoir lieu de deux manières, soit qu'ils naissent toujours à peu près en même nombre et meurent simplement après avoir fécondé les femelles destinées à persister, soit que le nombre des éclosions de mâles se trouve ralenti par suite d'un mécanisme qu'il reste à déterminer. Ce qui porte à penser que cette seconde hypothèse a quelque fondement, ce sont des faits dans le genre de celui qui est signalé par Mühlens et Sfarcic (1925) dans leur étude sur la campagne antipaludique de Dalmatie en 1924. Ces savants ont trouvé que le rapport des éclosions d'anophèles dans les élevages entrepris avec les œufs et les larves, était d'un mâle pour deux à quatre femelles. Or, ce chiffre me parait remarquablement faible, car dans les élevages que je fais depuis de nombreuses années, aux mois d'août et de septembre, en partant de larves ou de nymphes, j'ai toujours obtenu un fort excédent de mâles, surtout au début de l'élevage. L'éducation des nymphes constitue même un bon moyen de se procurer des mâles, car ce sont surtout des femelles qu'on trouve généralement dans la nature. On peut donc penser que Mühlens et Sfarcic ont opéré à tin moment où la production des mâles était ralentie.

Annales de Parasitologie, T. IV, $\mathrm{N}^{\circ} 2$. $-1^{\text {er }}$ avril 1926, p. 126-135. 
Cette formation des mâles peut être contrôlée et prévue par l'examen des larves, car il existe un moyen facile et certain de connaître leur sexe.

La plus ancienne indication bibliographique que j'aie relevée à ce sujet est une courte note d'H. Adie (1912), parue dans un périodique peu connu, édité à Simla de 1910 à 1912, par le Comité pour l'étude du paludisme dans l'Inde. Il semble que personne n'ait remarqué ce petit travail d'une vingtaine de lignes. Je me souviens très bien avoir sur le moment vérifié sur des larves de moustiques de la région parisienne, l'exactitude des observations d'Adie. Quelques années plus tard (1918), en décrivant la larve de l'Anopheles multicolor, j'ai signalé et figuré, dans le $6^{\circ}$ segment, les organes mentionnés en 1912 par Adie.

Voici la traduction in extenso de sa Note sur la distinction du sexe aux stades larvaire et nymphal des anophélines :

Si vous examinez une larve d'Anopheles avec une forte loupe, de $2 / 3$ de pouce, vous pouvez voir chez les mâles, de chaque côté du $6^{\circ}$ segment abdominal, une tuméfaction brune ovale. Avec de la pratique, on peut la reconnaître aisément avec une forte loupe dans une larve bien développée et même sur une larve de trois jours on peut la voir avec la loupe de 2/3. Dans les larves plus âgées, on peut voir aussi un tube dirigé vers la partie postérieure. Par la dissection, on trouve qu'il est relié à une vésicule et à un canal. La tuméfaction brune ovale est le testicule piriforme entouré de son sac épais et par la pression on trouve qu'il renferme des spermatozoïdes bien développés et rudimentaires. Ceci caractérise les mâles. Vingl-quatre pronostics se sont trouvés vérifiés par la métamorphose.

« Les ovaires et les spermathèques sont, à la dissection, tout à fait nets dans la nymphe femelle ainsi que ce que je considère comme les ovaires rudimentaires dans la larve femelle.

" Au stade nymphal, ces différences sont très voisines de celles qu'on trouve chez l'adulte.

“Dans le cas de larves de Culex, le testicule n'a pas la couleur brune si remarquable, ni le sac résistant et par conséquent n'est pas aussi reconnaissable ».

Je puis confirmer en grande partie les observations de Adie, car j'ai retrouvé les organes en question, non seulement chez les Anophélinés, mais aussi chez les Culicinés. J'ai vérifié que toujours, sans aucune exception, les larves de moustiques présentant ces organes donnent naissance à des individus mâles. Ces organes étant très pigmentés, sont faciles à voir par transparence sur la larve vivante et ils apparaissent encore plus nettement lorsqu'elle est

AnNales de Parasitologie, T. IV, No $2 .-1^{\text {er }}$ avril 1926. 
rendue transparente par le montage dans une résine (baume du Canada ou résine mastic).
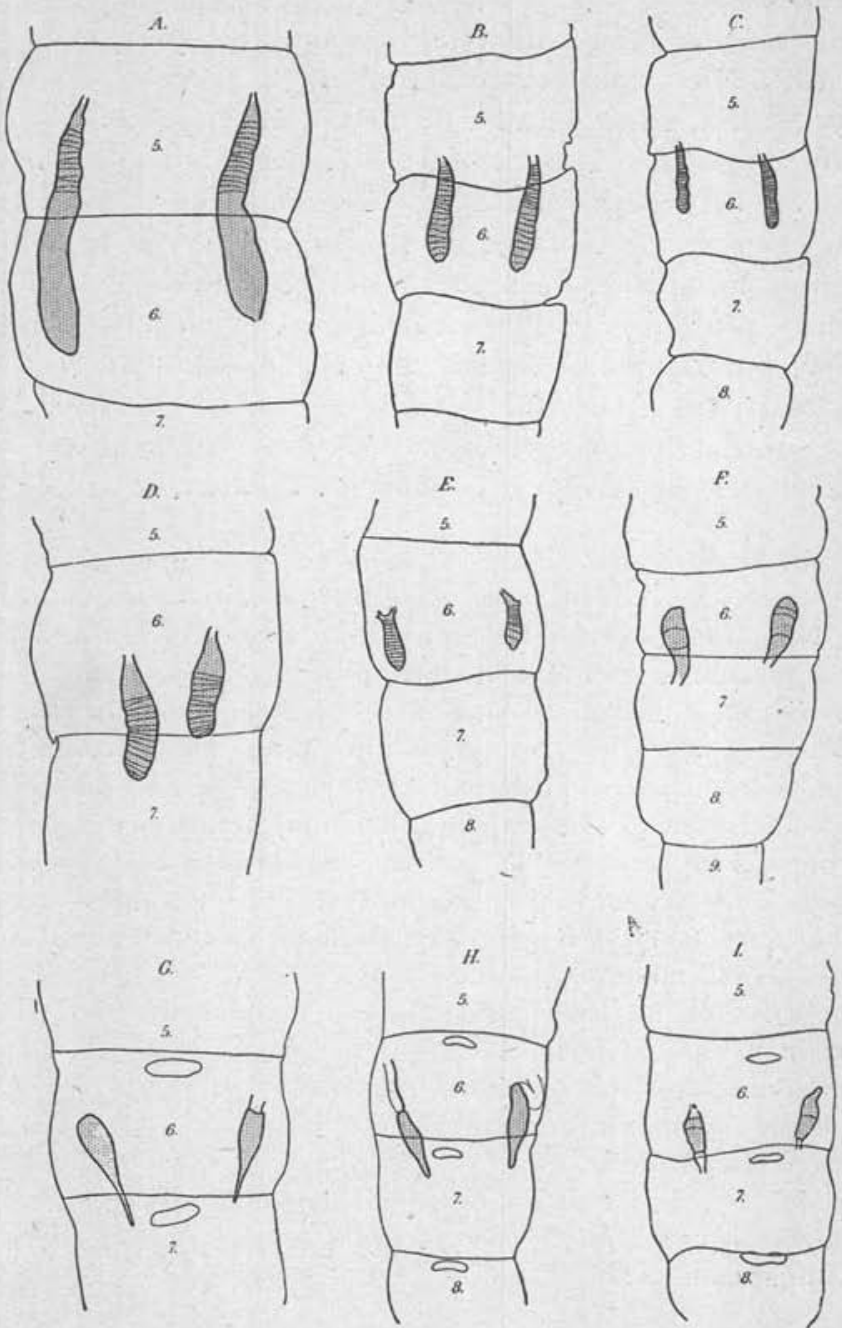

Fig. 1. - Morphologie comparée des testicules des larves mâles de divers moustiques. Segments abdominaux 5-7. - A, B, C, Theobaldia longiareolata à divers stades; D, Ochlerotatus dorsalis ; E, Culex hortensis ; F. I, Anopheles multicolor ; G, Anopheles maculipennis; H, Anopheles superpictus.

Ils sont situés, comme l'a vu Adie, au niveau du $6^{\circ}$ segment abdominal. Leurs connexions sont assez difficiles à établir. Elles parais- 
sent différentes suivant qu'il s'agit des larves à siphon des Culicinés, ou des larves sans siphon des Anophélinés.

Chez ces dernières, par exemple chez les larves mâles d'Anopheles maculipennis, la disposition est conforme à celle qui a été décrite par Adie. L'organe est de forme plus ou moins allongée (fig. 1, G) ; il est quelquefois renflé à sa partie médiane. Sa partie
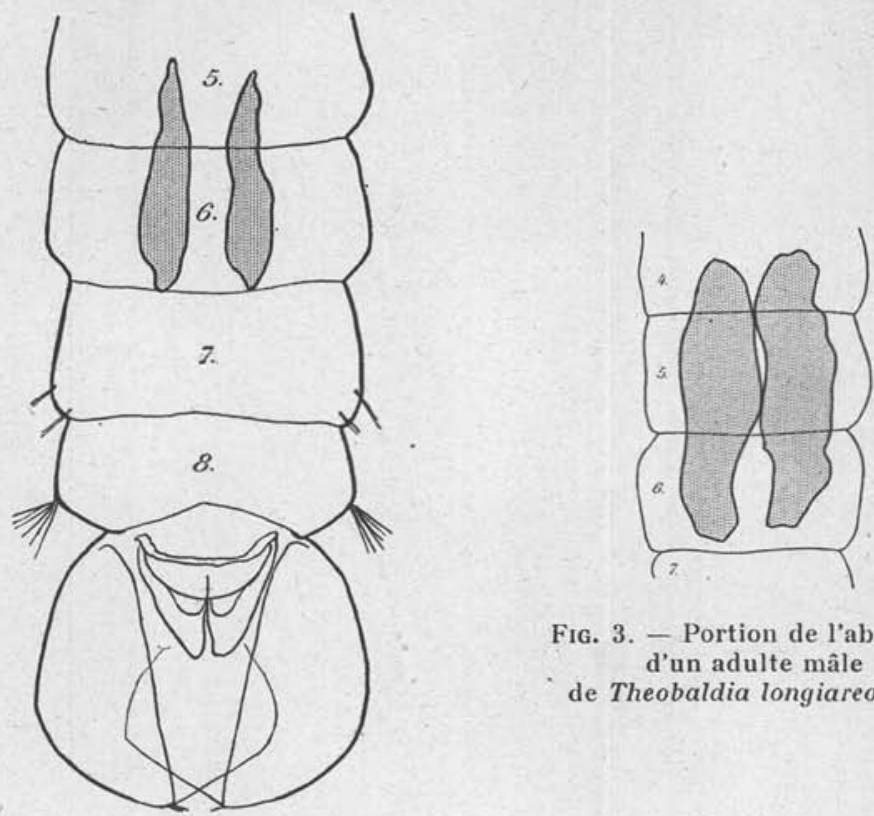

Fig. 3. - Portion de l'abdomen d'un adulte mâle de Theobaldia longiareolata.

Fig. 2. - Portion de l'abdomen de nymphe mâle

de Theobaldia longiareolata.

supérieure, qui ne dépasse généralement pas le $6^{\circ}$ segment, est brièvement atténuée et souvent mal délimitée; elle est presque toujours attachée à un tractus incolore fixé à la paroi latérale du $6^{\circ}$ segment. L'extrémité inférieure, qui pénètre souvent dans le $7^{\circ}$ segment, s'ètire en un étroit canal, plus ou moins allongé, quelquefois longuement effilé et qui se perd parmi les tubes de Malpighi.

Chez les Culicinés que j'ai pu étudier, j'ai observé une disposition inverse. Par exemple, chez Theobaldia longiareolata (fig. 1, A, B, C), l'extrémité de l'organe tournée vers le $7^{\mathrm{C}}$ segment se termine en cul-de-sac arrondi, tandis que l'extrémité supérieure est atténuée en un canal court. La même disposition se retrouve chez Culex hor- 
tensis (fig. 1, E), avec cette différence que l'extrémité supérieure paraît souvent bifurquée. Dans d'autres cas, les deux extrémités semblent se terminer par un canal.

Sur les coupes (fig. 5, 6, 7), ces organes apparaissent comme des

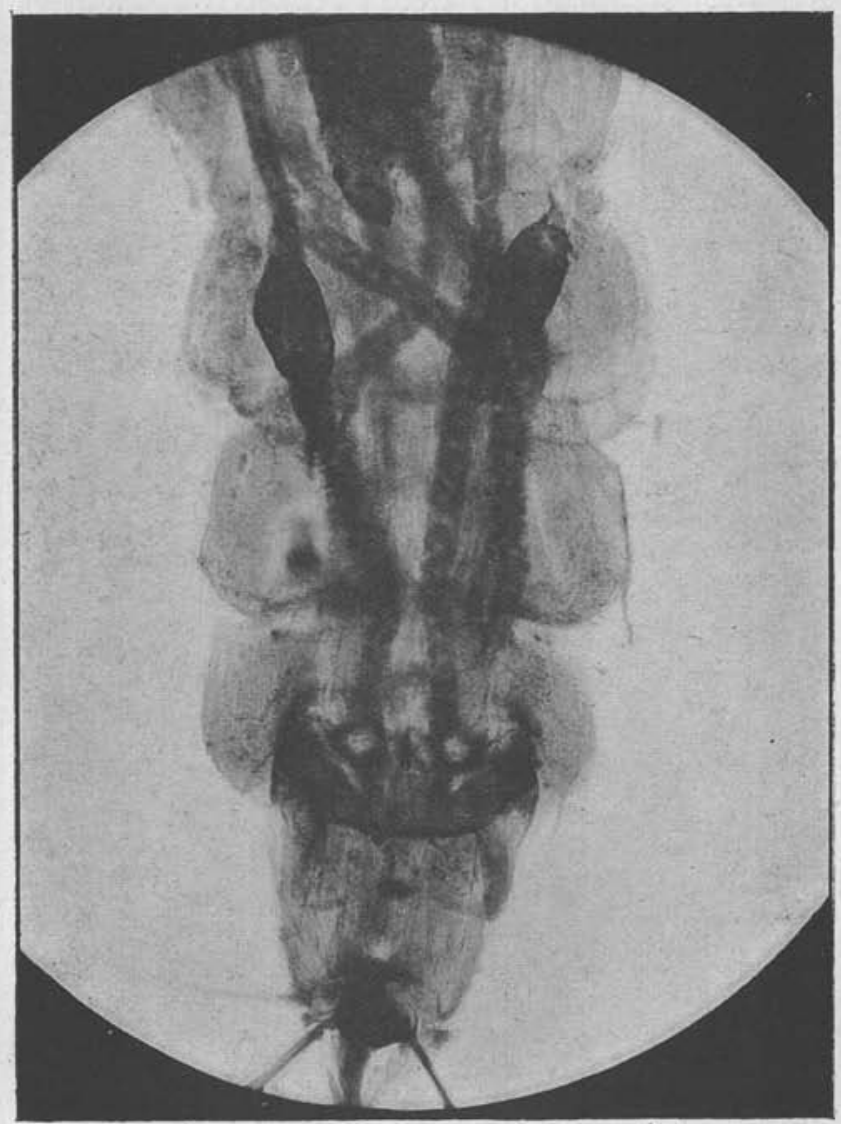

Fig. 4. - Abdomen de larve mâle d'Anopheles hispaniola, segments 5-9, montrant les testicules et les tubes de Malpighi.

sacs pigmentés. La paroi, assez épaisse, est formée de deux couches : la couche interne est composée de cellules très aplaties, à cytoplasme mince et à noyau volumineux et bombé.

La couche externe, très épaisse, est formée de volumineuses cellules, chargées de grains de pigment. Les contours de ces cellules 
sont difficilement visibles, mais on apërçoit très bien leur noyau (fig. 6).

La structure interne présente, comme la morphologie externe, des différences suivant qu'on étudie les Anophélinés ou les Culicinés. Chez Anopheles maculipennis (fig. 6), la cavité est unique, tandis que chez Theobaldia longiareolata (fig. 5 et 7 ), elle est cloi-

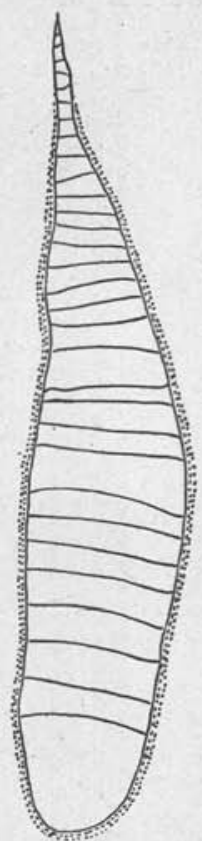

Fig. 5. - Testicule de larve mâle de Theobaldia longiareolata, figure demischématique montrant la paroi externe pigmentée et le cloisonnement.

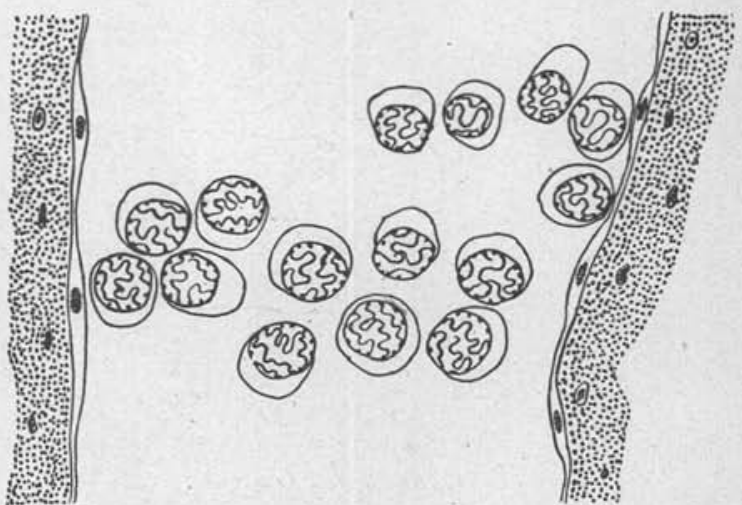

FIG. 6. - Portion d'une coupe du testicule d'une larve mâle d'Anopheles maculipennis, montrant les deux assises de la paroi et la strueture du noyau des cellules centrales.

sonnée. Les cloisons sont alors formées aux dépens du cytoplasme des cellules pariétales dont le noyau se trouve au point d'intersection de ces cloisons et de la paroi générale du sac.

Les larves d'Anopheles bifurcatus, A. algeriensis, A. sergenti, A. hispaniola (fig. 4), A. superpictus (fig. 1, H), présentent la même structure que celles d'A. maculipennis. Au contraire, chez A. multicolor (fig. 1, F, I), le testicule est nettement cloisonné, mais les cloisons sont peu nombreuses, et ne forment que deux ou trois loges. Le contenu du sac est formé de cellules à noyaux volumineux, 
vésiculeux, à contenu incolore et à filament chromatique sinueux, ressemblant à un ruban appliqué à la surface (fig. 6). Les coupes optiques de ce ruban apparaissent comme autant de points chromatiques à la périphérie du noyau, mais la manœuvre de la vis micrométrique renseigne de suite sur leur véritable nature. Le cytoplasme est très peu développé.

L'état des noyaux varie de la base au sommet de l'organe et les

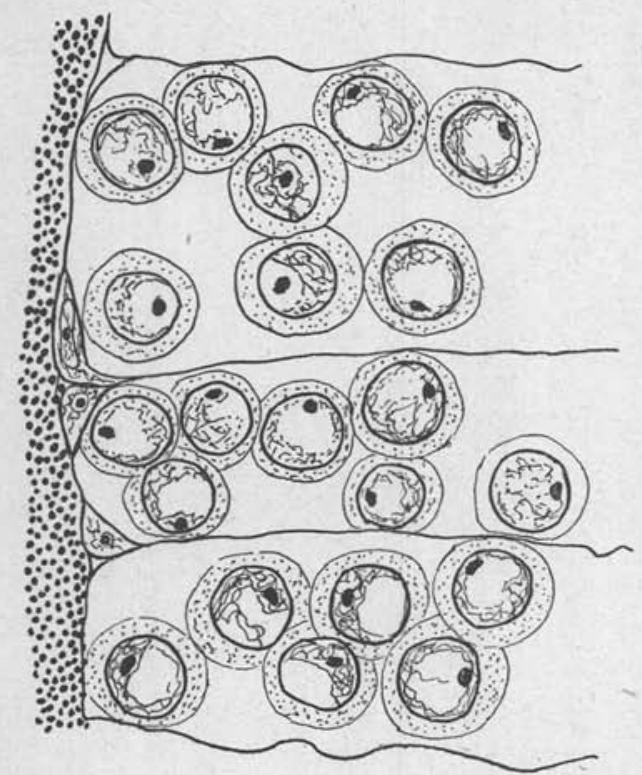

Fig. 7. - Portion d'une coupe du testicule d'une larve mâle de Theobaldia tongiareolata, montrant le cloisonnement.

variations sont parallèles chez les Culicinés et les Anophélinés.

A la base, c'est-à-dire du côté du $7^{\circ}$ segment, les noyaux possèdent la structure que je viens de décrire. Chez Theobaldia longiareolata (fig. 7 ), le nucléole est très apparent et se colore en rose vif par le Mann biacide. Cette structure persiste dans les deux tiers de la hauteur de l'organe. Vers le haut, c'est-à-dire dans la direction du $5^{\circ}$ segment, l'aspect des noyaux change. Ils sortent brusquement de l'état quiescent: le filament chromatique se pelotonne en une masse compacte, les chromosomes se constituent, puis des figures de caryocinèse apparaissent. Dans mes coupes de larves de Theolaldia longiareolata, ces figures sont très nettes : on voit des plaques équatoriales avec le double fuseau achromatique très apparent 
(fig. 8). Une de ces coupes intéresse exactement l'extrémité effilée de l'organe mâle (fig. 5). Au contraire, dans son matériel d'Anopheles maculipennis, peut-être moins bien fixé, les figures sont plus confuses. Ce sont plutôt des pelotons et des amphiasters.

Telles sont les observations qu'on peut faire en examinant des larves au $4^{*}$ stade, soit montées en entier dans une résine, soit débitées en coupes sériées après fixation au Duboseq-Brasil, inclusion à la paraffine et coloration à l'hémalun-éosine ou au Mann biacide.

Comme l'a indiqué Adie, les mêmes organes se retrouvent chez les nymphes, dans lesquelles on les voit facilement par transparence (fig. 2).

Ces organes apparaissent de très bonne heure, car on les trouve

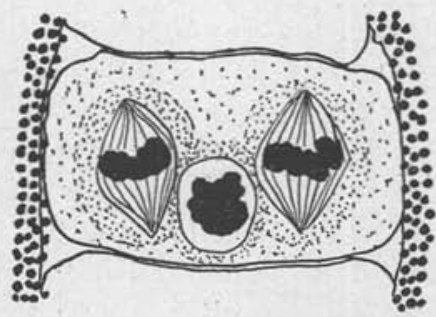

Fig. 8. - Figures de caryocinèse dans la partie supérieure du testicule d'une larve mâle de Theobaldia longiareolata.

chez de très jeunes larves, encore au $2^{\circ}$ stade. Naturellement ils sont moins pigmentés et leurs contours sont moins nets, mais on les aperçoit à leur place, au niveau du $6^{\circ}$ segment abdominal. On peut donc faire un triage très précoce des larves mâles, en les examinant individuellement au microscope, entre lame et lamelle, et suivre le développement de l'organe pigmenté.

Chez les adultes mâles, on retrouve ces organes à la même place, comme le montre la fig. 3, représentant une partie de l'abdomen d'un mâle de Theobaldia longiareolata. Ils empiètent seulement un peu sur les segments inférieur et supérieur, surtout dans les types où ils sont très volumineux.

Dans un travail ultérieur, je ferai connaître leur structure chez les adultes et les différences qu'elle présente avec l'état larvaire.

Il est intèressant de noter la persistance de ces organes à travers les métamorphoses des moustiques et malgré les phénomènes d'histolyse. Ce n'est pas un des problèmes les moins intéressants que soulève leur étude. 
Pour déterminer le rapport entre l'anophélisme résiduel et le sexe des larves, j'ai examiné au point de vue de leur sexualité les larves d'Anopheles pêchées en Corse en août-octobre 1925, avec les $\mathrm{D}^{\mathrm{rs}}$ Larrousse et Galliard, pour la Station d'application antipaludique de Bastia. Voici les chiffres que j'ai trouvés :

Sur 34 larves d'Anopheles algeriensis, il y avait 31 larves femelles et trois larves mâles, ce qui correspond bien avec la disparition précoce de cette espèce en automne.

Sur 269 larves d'Anopheles maculipennis, il y avait 88 larves mâles et 181 larves femelles, ce qui donne à peu près un mâle pour deux femelles. Le rapport varie d'ailleurs avec la saison. C'est ainsi que le 15 août 1925 nous trouvons à la briqueterie de la Fornacina, près de Bastia, trois larves mâles pour trois femelles, les adultes capturés en même temps dans une écurie de chevaux comprenant deux mâles pour 21 femelles d'Anopheles maculipennis. Le 24 août, à Cateraggio, nous trouvons 4 larves mâles d'Anopheles maculipennis pour 7 femelles et le 26 août sur 21 adultes d'Anopheles maculipennis capturés dans un poulailler de la même localité, il y avait 21 femelles. Le 14 octobre, à l'étang del Sale, nous ne trouvons plus que 5 larves małles pour 16 femelles, soit $1: 3$. Cette diminution correspond à l'apparition de l'anophélisme résiduel et à l'absence de mâles dans les captures d'adultes.

Pour l'Anopheles bifurcatus qui apparaît plus tardivement, persiste plus longtemps et hiverne à l'état larvaire, je trouve, sur 94 larves, 40 mâles et 54 femelles, ce qui donne un peu moins d'un mâle pour une femelle, soit un peu plus de $4 / 5$ de larves mâles.

Bien que ces chiffres ne portent que sur un petit nombre d'observations, ils montrent que la diminution du nombre des larves mâles accompagne l'apparition de l'anophélisme résiduel.

Je pensais que la morphologie de l'organe mâle pourrait être de quelque utilité pour la détermination des larves. Il n'en est rien puisque des larves d'espèces très différentes comme $A$. maculipennis, A. bifurcatus, A. algeriensis, A. hispaniola, A. sergenti, A. superpictus, présentent à ce point de vue des caractères presque identiques. Mais ces données pourront être utilisées pour établir des coupures dans le genre Anopheles. Les anciens genres résultant de son démembrement ont été ramenés au rang de sous-genres, ce qui, à mon avis, n'a pas apporté beaucoup de clarté et présente le grand inconvénient de nous conduire insidieusement à la nomenclature trinominale. En utilisant des caractères tels que ceux que présente l'organe mâle, on pourra peut-être rétablir de bonnes coupures dans le genre Anopheles, trop compréhensif, et en détacher 
des genres bien caractérisés par ce détail anatomique. Il est certain, par exemple, que le testicule d'A. multicolor est très différent de celui d'autres Anopheles paléarctiques. Des études comparées, entreprises avec un matériel suffisant, permettront de décider s'il y a lieu de démembrer de nouveau ce genre. Les mêmes résultats pourront être obtenus chez les Culicinés.

\section{RÉSUMÉ}

Les testicules signalés par Adie en 1912 chez des larves d'Anopheles de l'Inde, au niveau du $6^{\circ}$ segment abdominal, ont été retrouvés chez les larves d'un certain nombre de moustiques de France et du bassin méditerranéen. Ces organes sont visibles de très bonne heure chez la jeune larve. Leur forme et leur structure diffèrent chez les anophélinés et les culicinés, ainsi qu'entre les divers genres de ces deux sous-familles.

La facilité avec laquelle ces organes sont vus par transparence permet de reconnaître rapidement le sexe des larves et de contrôler l'évolution saisonnière des espèces dans ses rapports avec l'anophélisme résiduel.

\section{BIBLIOGRAPHIE}

ADIE (H.-A.). - Distinction of sexe in the larval and pupal stages of Anophelines. Paludism, sept. 1912, n० 5, p. 41.

Langeron (M.). - La larve d'Anopheles Chandoyei (Theobald, 1903). Bull. Soc. pathol. exotique, XI, 1918, p. 291-297 ; cf. p. 296, fig. 5.

Mühlens (P.) et SFARcic (A.). - Bericht über Malariaarbeiten in Dalmatien. Centralblatt für Bakteriologie, Orig., XCIV, 1925, p. $326-376,3$ pl.

Swellengrebel (N.-H.). - A summary of the more important facts on adult anophelines and their larvæ .. D. Residual anophelism. League of nations. Health organisation. Malaria commission. Report on its tour of investigation in certain european countries in 1924. C. H. 273. Genève, 1925, p. 172-190; cf. p. 181.

Laboraloire de Parasitologie dé la Faculté de médecine de Paris 